14,19

\title{
Фазовые и релаксационные переходы в политетрафторэтилене
}

\author{
(C) В.М. Егоров, П.Н. Якушев \\ Физико-технический институт им. А.Ф. Иофрфе РАН, \\ Санкт-Петербург, Россия \\ E-mail: victor_egorov1@inbox.ru
}

(Поступила в Редакцию 12 марта 2018 г.)

Проведен сравнительный анализ фазовых и релаксационных переходов в политетрафторэтилене методами дифференциальной сканирующей калориметрии и динамического механического анализа. Устранение методических погрешностей в первом случае позволило получить истинные значения термодинамических параметров фазовых переходов и выявить их природу. Проведен количественный анализ профиля пика теплоемкости на основе теории размытых фазовых переходов первого рода, а также охарактеризован релаксационный переход стеклования.

DOI: $10.21883 /$ FTT.2018.09.46405.060

Выяснение природы и эволюции фазовых (ФП) и релаксационных переходов (РП) в твердых телах, включая полимеры, составляет один из важнейших разделов физики твердого тела. Оба типа переходов в полимерах обычное явление, т.к. большинство из них находится в двухфазном состоянии - частично кристаллическом, в котором наблюдаются термодинамические фазовые переходы, и аморфном (термодинамически - жидком), в котором наблюдаются релаксационные переходы. Оба типа переходов могут быть инициированы различными внешними воздействиями, включая температуру, механические напряжения, гидростатическое давление, электрические и магнитные поля, то есть реализуются практически во всех процессах получения и переработки полимерных материалов.

Значительная дисперсия характеристик полимеров как на молекулярном уровне, так и на различных иерархических уровнях надмолекулярной организации приводит к заметному размытию параметров, характеризующих $Ф П$. Это существенно затрудняет выявление природы ФП в кристаллической части полимеров и затрудняет анализ экспериментальных данных на основе имеющихся в настоящее время теорий фазовых переходов I и II рода.

Не менее важными с точки зрения понимания механизма молекулярной подвижности в полимерах и их практического применения являются так называемые релаксационные переходы. Основной релаксационный переход в полимерах - $\alpha$-переход стеклования, в температурном интервале которого происходит наиболее кардинальные изменения физических свойств полимеров. Явление стеклования (размягчения) рассматривают в основном на языке кинетических представлений [1-3]. Однако в теории существуют предположения о существовании термодинамического ФП, ответственного за проявления релаксационного перехода стеклования. Этот переход должен предшествовать по температуре реально наблюдаемой температуре стеклования $\left(T_{g}\right)$ и являться температурным пределом для нее [4-7].
В настоящей работе исследуется политетрафторэтилен (ПТФЭ), в котором наблюдается сочетание ФП и РП. Наряду с традиционными для полимеров (стеклование, т.н. „вторичный“ $\beta$-переход), в нем, помимо плавления, наблюдаются твердотельные фазовые переходы. В качестве образцов для исследования использовался промышленный ПТФЭ марки Ф-4Д производства OAО „Галоген“. Динамический механический анализ проводился на модуле DMS6100 рабочей станции EXSTAR6000 фирмы Seiko Instruments. Образцы в виде цилиндров диаметром $3 \mathrm{~mm}$ и длиной $6 \mathrm{~mm}$ испытывались в режиме синусоидального приложения нагрузки сжатия с частотой $1 \mathrm{~Hz}$ при нагревании со скоростью $2 \mathrm{~K} / \mathrm{min}$ в том же интервале температур, что и в методе дифференциальной сканирующей калориметрии (ДСК). На рис. 1 представлены полученные в работе зависимости $\tan \delta=F(T)$. Наибольшие динамические потери, проявляющиеся в виде пиков на кривой $\tan \delta=F(T)$, наблюдаются в температурных диапазонах как фазовых

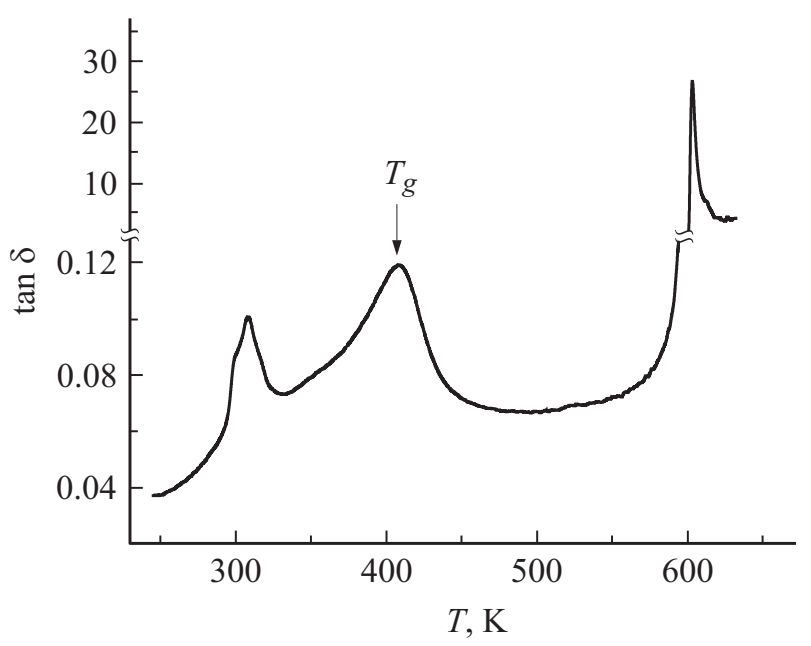

Рис. 1. Зависимость величины динамических потерь $(\tan \delta)$ ПТФЭ от температуры. 


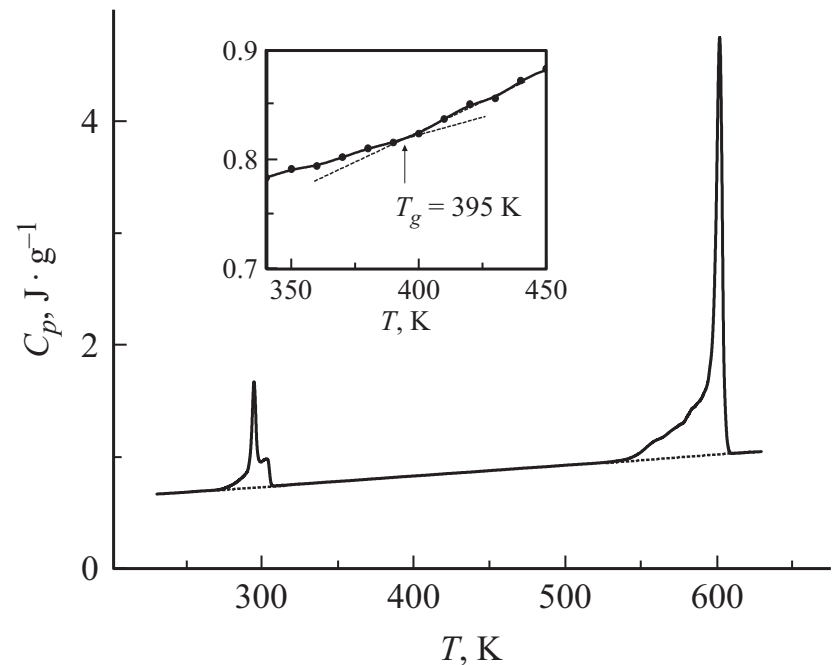

Рис. 2. Зависимость теплоемкости $C_{p}$ ПТФЭ от температуры.

переходов - твердотельного $(\sim 300 \mathrm{~K})$ и плавления $(\sim 600 \mathrm{~K})$, так и релаксационного перехода стеклования ( 400 K).

В отличие от данных по динамическим потерям, полученным при активном воздействии на полимер механической нагрузки, на зависимости теплоемкости от температуры $C_{p}(T)$ (рис. 2) релаксационный переход стеклования при $T=395 \mathrm{~K}$ выражен очень слабо (см. вкладку к рис. 2). На зависимости $C_{p}(T)$ четко различимы только эндотермические пики, отвечающие твердофазному переходу (дублет при $\sim 300 \mathrm{~K})$ и плавлению $(\sim 600 \mathrm{~K})$. Обусловлено это, по-видимому, тем, что сегментальное движение в ПТФЭ затруднено жесткостью цепи, статистический сегмент которой $\left(\sim 18\right.$ групп $\left.-\mathrm{CF}_{2}-\right)$ аномально высок для гибкоцепных полимеров. Поэтому для проявления кооперативного движения сегментов, характерного для стеклования, необходимо внешнее воздействие, в данном случае, механическим полем.

По площади пиков на зависимости $C_{p}(T)$ определены энтальпия $(\Delta H)$ и энтропия $(\Delta S)$ переходов, которые в методе ДСК не зависят от скорости сканирования по температуре и определяются по соотношениям $\Delta H_{\exp }=\int C_{p}(T) d T$ и $\Delta S_{\exp }=\int C_{p}(T) d(\ln T)$. Значения этих параметров для твердофазного ФП составили $\Delta H_{\exp }=8.5 \mathrm{Jg}^{-1}$ и $S_{\exp }=0.028 \mathrm{Jg}^{-1} \mathrm{~K}^{-1}$. Степень кристалличности $(\chi)$, определенная по соотношению $\chi=100 \Delta H_{m} / \Delta H_{m}^{0}$, где $H_{m}=35 \mathrm{Jg}^{-1}-$ экспериментально измеренная энтальпия плавления; $\Delta H_{m}^{0}=69 \mathrm{Jg}^{-1}$ - энтальпия плавления совершенного кристалла ПТФЭ [8], оказалась равна 51\%. Дополнительные калориметрические измерения теплоемкости, приведенные далее, позволили охарактеризовать эндотермический дублет в температурном диапазоне 230-330 K как сумму твердофазных термодинамических переходов первого и второго рода. Однако перед изложением этих данных необходимо сделать предварительное замечание.
Во многих опубликованных работах приводятся данные по температурным характеристикам тех или иных экзо- и эндо-эффектов, в которых не были устранены методические погрешности, связанные с эффектом термосопротивления при ДСК измерениях. Это может приводить к существенным искажениям значений термодинамических характеристик изучаемых материалов. Кроме того, как правило не проводятся и не анализируются ДСК измерения в циклах нагревание-охлаждение, которые, как будет показано в данной работе, позволяют выявить природу фазовых переходов.

На рис. 3 в качестве примера приведены кривые ДСК, полученные при нагревании и охлаждении образцов ПТФЭ при одной скорости сканирования $V=1.25 \mathrm{~K} \cdot \mathrm{min}^{-1}$. Эти кривые показывают относительное изменение теплоемкости в температурном интервале переходов $\Delta C_{p}(T)=C_{p}(T)-C_{\text {ref, }}$ где $C_{p}(T)-$ теплоемкость образца (см. рис. 2); $C_{\text {ref }}$ - теплоемкость эталона, экстраполируемая линейной зависимостью $C_{\text {ref }}=a \cdot T+b$, при значениях $a=9.58 \cdot 10^{-4}$ и $b=0.45$. При нагревании на кривых видны эндотермические, а при охлаждении - экзотермические пики твердотельного ФП.

Видно, что процесс изменения фазового состояния происходит в два этапа (температуры максимумов и минимумов соответствующих им эндотермических и экзотермических пиков $T_{\max 1}, T_{\max 2}$ и $T_{\min 1}, T_{\min 2}$ ). Для понимания физической природы регистрируемых на термограммах переходов необходимо прояснить картину с наблюдаемым температурным несовпадением пиков: на кривых ДСК видны смещения относительных положений максимумов эндо- и экзо-пиков в циклах нагревание-охлаждение $\left\{\left(T_{\max 1}-T_{\min 1}\right)\right.$ и $\left.\left(T_{\max 2}-T_{\min 2}\right)\right\}$. Оказалось, что смещения (так называ-

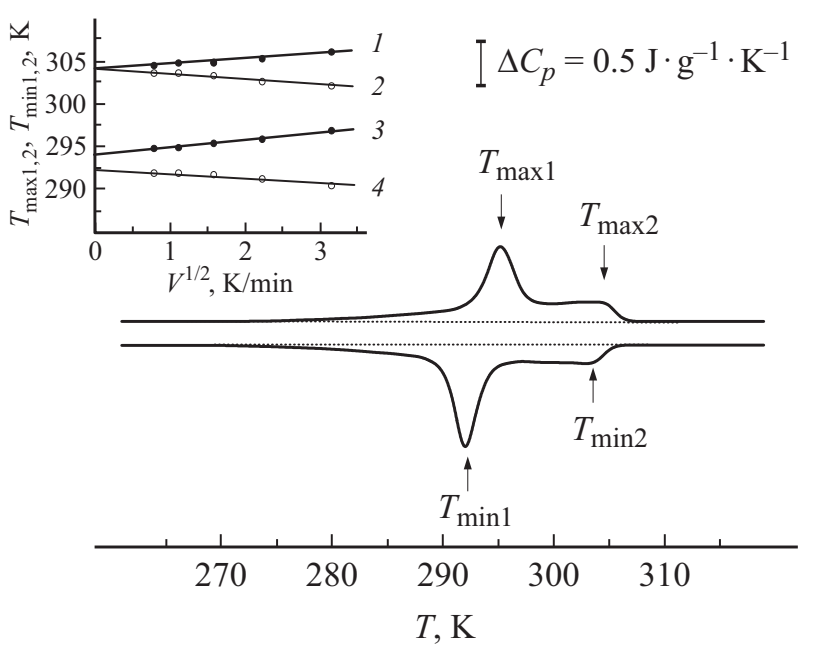

Рис. 3. Кривые ДСК нагревания и охлаждения ПТФЭ; скорость сканирования $V=1.25 \mathrm{~K} / \mathrm{min}$. На вставке - зависимости температур $T_{\max 1}$ (3), $T_{\max 2}(1), T_{\min 1}$ (4) и $T_{\min 2}$ (2) твердофазных переходов от скорости нагревания $(1,3)$ и охлаждения $(2,4)$. 
емый эффект гистерезиса) имеют для каждого из пиков дублета свою природу, обусловленную как методической, так и физической причинами.

Известно [9], что при использовании метода ДСК возникают методические погрешности (смещение пиков), связанные с наличием термосопротивления испытываемого образца в калориметрической ячейке, которое существенно зависит от массы образца и скорости сканирования. В работе [10] показано, что эти методические погрешности могут быть устранены следующим образом: по экспериментальным данным при вариации скоростей сканирования $V$ (нагревания или охлаждения) строятся зависимости $T_{\max , \min }=f\left(V^{1 / 2}\right)$, которые при отсутствии каких-либо структурных трансформаций должны быть линейными. Экстраполяция линейных зависимостей к $V \rightarrow 0$ позволяет получить истинные, методически неискаженные значения температур переходов. В настоящей работе были получены подобные зависимости в циклах нагревание-охлаждение (см. вставку к рис. 3) и определены экстраполяционные (истинные значения) температур фазовых переходов $T_{\max 1}=294.3 \pm 0.2 \mathrm{~K}$ и $T_{\min 1}=292.3 \pm 0.2 \mathrm{~K}$, а также $T_{\max 2}=T_{\min 2}=304.4 \pm 0.2 \mathrm{~K}$. Величина температурного гистерезиса для более низкотемпературного пика составила $\Delta T=T_{\max 1}-T_{\min 1}=2 \pm 0.2 \mathrm{~K}$.

На основании этих данных можно заключить, что в действительности для высокотемпературных максимумов температурный гистерезис в циклах нагревание-охлаждение либо отсутствует, либо представляет собой незначительную величину $(\Delta T<0.2 \mathrm{~K})$, а наблюдаемая на экспериментальных кривых ДСК разница в их положении на температурной шкале обусловлена методическими причинами. В то же время даже после устранения методической погрешности в этих образцах наблюдается эффект гистерезиса для низкотемпературного перехода.

Как известно [11], наличие или отсутствие температурного гистерезиса какого-либо физического свойства, в том числе для пиков на температурной зависимости теплоемкости, является одним из признаков, по которому фазовые переходы относят соответственно к переходам первого или второго рода. Поэтому отсутствие гистерезиса для вторых максимумов прямо указывает на принадлежность высокотемпературных пиков в дублете к переходам второго рода. Если не использовать экстраполяционные зависимости $T_{\max , \min }=f\left(V^{1 / 2}\right)$, применяемые в настоящей работе, а пользоваться лишь данными, полученными при одной скорости сканирования по температуре, то высокотемпературные пики можно ошибочно также отнести к переходам первого рода. С другой стороны, наличие истинного гистерезиса для низкотемпературного пика позволяет на основании данных ДСК отнести этот переход к твердофазному (структурному) переходу I рода.

Ранее $[8,12]$, методом рентгеновской дифракции было показано, что в кристаллической части ПТФЭ при нагревании происходит фазовый переход первого рода

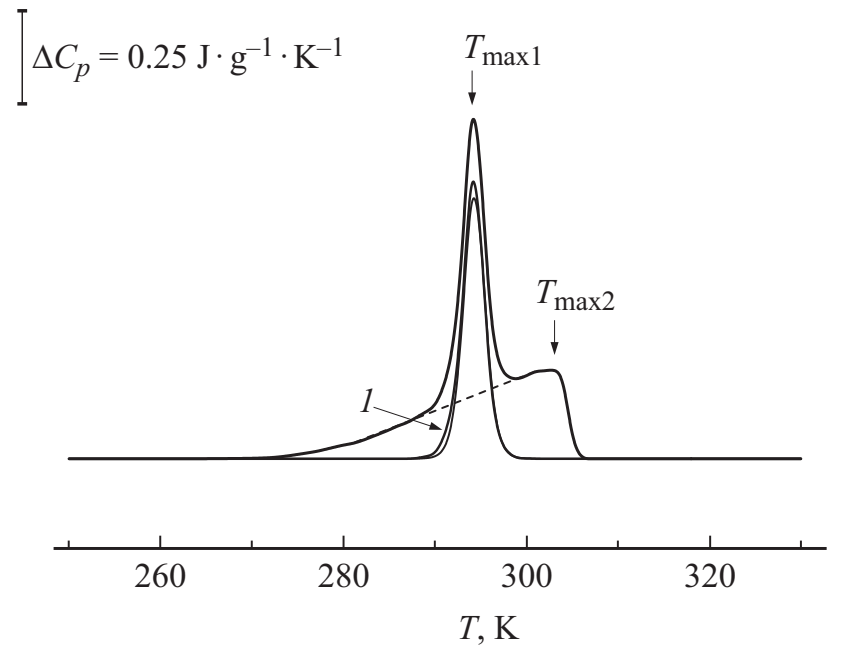

Рис. 4. Эндотермические пики, отвечающие твердофазным переходам. Жирные линии - экспериментальные данные, тонкая линия - результат расчета по зависимости (1).

при $T_{\max } 1$ из структуры с триклинной элементарной ячейкой в структуру, поперечная упаковка молекул в которой близка к гексагональной. При температурах выше $T_{\max 1}$, вплоть до температуры плавления, эта поперечная упаковка молекул сохраняется, то есть сохраняется дальний порядок. Такое строение структуры можно охарактеризовать как жидкокристаллическое.

Важная дополнительная информация о физической природе этого фазового перехода может быть получена при анализе формы пика теплоемкости на основе теории самосогласованного поля применительно к $\Lambda$-образным размытым переходам I рода [13-15].

На рис. 4 пик теплоемкости ФП первого рода выделен из дублета путем линейной экстраполяции, связывающей низкотемпературную и более высокотемпературную части ФП второго рода. Прямая, отвечающая этой экстраполяции, обозначена штриховой линией. Выделенный таким образом пик по форме напоминает так называемый $\Lambda$-образный пик (кривая 1 ).

Тот факт, что переход становится размытым, означает, что изменение фазового состояния происходит не внезапно, но постепенно, и осуществляется в пределах определенного температурного интервала. Образование зародышей-доменов новой фазы объемом $\omega$ требует изменения температуры на малую величину, которая определяется энергией, необходимой на возникновение таких областей. Это и приводит к размытию перехода по температуре. Например, для сегнетоэлектрических материалов было показано, что элементарные объемы превращения $\omega$ сопоставимы с объемом так называемой области Кенцига $\left(\sim 10^{-18}-10^{-17} \mathrm{~cm}^{3}\right)$ и по своему масштабу находятся на мезоскопическом уровне [16,17].

Оказалось, что размеры устойчивых зародышей $\omega$ можно определить из формы пиков теплоемкости, соответствующих переходам I рода. В работе [18] получено 
соотношение для температурной зависимости теплоемкости при размытом фазовом переходе в виде

$$
\begin{aligned}
\Delta C_{p}(T)= & 4 \Delta C_{m} \cdot \exp \left[B\left(T-T_{0}\right) / T_{0}\right] \\
& \times\left[1+\exp \left[B\left(T-T_{0}\right) / T_{0}\right]\right]^{-2},
\end{aligned}
$$

где $T_{0}$ - температура фазового перехода первого рода; $\Delta C_{m}-$ максимальное значение теплоемкости при $T=T_{0} ; B-$ атермический параметр.

Расчет теплоемкости по соотношению (1) производился по значениям $T_{0}$ и $\Delta C_{\max }$, соответствующим температуре и амплитуде максимума пика. Результат расчета приведен на рис. 4 в виде тонкой линии. Наилучшее совпадение расчетных и экспериментальных зависимостей наблюдалось при значении параметра $B=450$.

Параметр $B$ в приведенном выше соотношении содержит наиболее интересную информацию о физической природе фазового перехода, поскольку он связан с величиной пика теплоемкости $\Delta C_{m}$ соотношением

$$
\Delta C_{m}=q_{0} B / 4 T_{0},
$$

где $q_{0}$ - теплота превращения, и элементарным объемом превращения $\omega$

$$
B=\omega \rho q_{0} / k T_{0},
$$

где $k-$ постоянная Больцмана, $\rho-$ плотность. Параметр $B$ оказывается структурно чувствительным параметром, поскольку определяет в материалах с размытыми фазовыми переходами объемы зародышей новой фазы. Из соотношений (2) и (3) был определен элементарный объем фазового превращения в кристаллической фазе полимера $\omega=185 \mathrm{~nm}^{3}$ (плотность кристаллов ПТФЭ $\left.\rho=2.2 \mathrm{~g} \cdot \mathrm{cm}^{-3}[19]\right)$. Оценка числа отрезков макромолекул, сопоставимых с размером статистического сегмента, участвующих в образовании зародыша новой фазы, показывает, что в элементарных объемах превращения их содержание составляет $\sim 15-16$ единиц, то есть область превращения захватывает весьма большой домен.

Возвращаясь к ФП второго рода, частично охарактеризованному выше, следует отметить, что он связан с потерей спиральной хиральности длинноцепочечной молекулы кристалла ПТФЭ, то есть с потерей элемента симметрии кристалла. Известно [8], что при повышении температуры в температурном диапазоне перехода спираль молекулы ПТФЭ с повторяющимся звеном из 6 витков и 13 групп - $\mathrm{CF}_{2}-$,„раскручивается“ и повторяющееся звено увеличивается до 7 витков и 15 групп $-\mathrm{CF}_{2}-$, приближаясь к размеру статистического клубка. Выше $\sim 303 \mathrm{~K}$ возрастает свобода внутренних вращений, вследствие чего определить наличие спирали и повторяющихся звеньев экспериментально определить не удается.

Потеря спиральной хиральности кристалла ПТФЭ приводит к увеличению конформационной энтропии на величину $\Delta S$. Этот процесс на молекулярном уровне можно рассматривать как переход элементарных независимых групп, состоящих из $Z$ звеньев $-\mathrm{CF}_{2}-$, из одного состояния в другое, то есть до и после перехода. Изменение конформационной энтропии для одного моля звеньев $-\mathrm{CF}_{2}-$ кристалла $(\mathrm{Na})$ тогда можно представить в виде соотношения $\Delta S=\left(N_{a} / Z\right) k \ln 2$ [7]. Исходя из экспериментально определенной энтропии фазового перехода II рода, равной $\Delta S_{\text {exp }}=0.016 \mathrm{~J} \cdot \mathrm{K}^{-1} \cdot \mathrm{g}$, можно оценить величину $Z$. Она оказалась равна $\sim 14-15$ звеньям $-\mathrm{CF}_{2}-$, то есть величиной, сопоставимой с периодом спирали молекулы ПТФЭ.

Обсуждаемый твердофазный переход II рода в ПТФЭ может активировать продолжение длительной дискуссии о термодинамической природе релаксационного перехода стеклования. Впервые стеклование как фазовый переход второго рода предложили рассматривать Гиббс и Ди Марцио [6]. По выдвинутой ими теории предполагается, что при приближении к температуре стеклования (при охлаждении) равновесным способом жидкость должна претерпеть фазовый переход второго рода, вследствие чего конфигурационная энтропия жидкости должна приблизиться к энтропии кристалла. Этот переход можно считать практически ненаблюдаемым, поскольку при приближении к температуре этого гипотетического перехода время релаксации экспоненциально растет и происходит переход в неравновесное стеклообразное состояние. Однако, если предположить, что при охлаждении удастся равновесно приблизиться к точке гипотетического перехода и конфигурационная энтропия жидкости вследствие этого перехода станет близкой конфигурационной энтропии кристалла, то нагревание такого кристалла должно сопровождаться вначале обратным фазовым переходом II рода при той же температуре, а затем, при дальнейшем нагревании, плавлением кристалла. В этом случае энтропия плавления должна содержать только конфигурационную часть, а ее изменение при плавлении будет определяться энтропией „смешения“ $\Delta S_{\text {mix }}=R \ln 2$, равной для моля звеньев $-\mathrm{CF}_{2}-\Delta S_{\text {mix }}=0.10 \mathrm{~J} \cdot \mathrm{g}^{-1} \mathrm{~K}^{-1}$, где $R=8.31 \mathrm{~J} \cdot \mathrm{mol}^{-1} \mathrm{~K}^{-1}$. Экспериментально определяемая энтропия плавления ПЭТФ составляет $\Delta S_{f}=0.115 \mathrm{~J} \cdot \mathrm{g}^{-1} \mathrm{~K}^{-1}$. Таким образом, оказывается, что бо́льшая часть энтропии плавления ПТФЭ составляет энтропия „смешения“, и предположение о том, что гипотетический фазовый переход стеклования может реально существовать, имеет реальное обоснование.

\section{Список литературы}

[1] П.П. Кобеко. Аморфные вещества. Изд-во. АН СССР, М.Л. (1952). $431 \mathrm{c}$.

[2] E. Donth. Glasubergang. Akademie Verlag, Berlin (1981). $202 \mathrm{p}$.

[3] О.В. Мазурин. Стеклование. Наука, Л. (1986). 156 с.

[4] J.H. Gibbs. J. Chem. Phys. 25, 185 (1956).

[5] J.H. Gibbs, E.A. DiMarzio. J. Chem. Phys. 28, 373 (1958). 
[6] E.A. DiMarzio, J.H. Gibbs. J. Chem. Phys. 28, 807 (1958).

[7] G.A. Adam, J.H. Gibbs. J. Chem. Phys. 43, 139 (1965).

[8] Б. Вундерлих. Физика макромолекул. Мир, М. (1984) Т. 3. $479 \mathrm{c}$.

[9] K. Illers. Eur. Polym. J. 10, 911 (1974).

[10] V.A. Bershtein, V.M. Egorov. Differential Scanning Calorimetry of Polymers: Physics, Chemistry, Analysis, Technology. Ellis Horwood, N.Y. (1994). 253 p.

[11] Л.Д. Ландау, Е.М. Лифшиц. Статистическая физика. Наука, М. (1976). $584 \mathrm{c}$.

[12] Polymer Handbook / Eds J. Brandrup, E. Immergut. Willey, N.Y. (1975). $452 \mathrm{p}$.

[13] М. Фишер. Природа критического состояния. Мир, М. (1973). $260 \mathrm{c}$

[14] Б.Н. Ролов, В.Э. Юркевич. Физика размытых фазовых переходов. Изд-во Рост. ун-та, Ростов (1983). 310 с.

[15] Г.А. Малыгин. Успехи физ. наук 171, 187 (2001).

[16] В. Кенциг. Сегнетоэлектрики и антисегнетоэлектрики. ИЛ, М. (1960). 324 с.

[17] В.Я. Фрицберг. Изв. АН СССР. Сер. физ. 47, 698 (1983).

[18] Г.А. Малыгин. ФТТ. 43, 1911 (2001).

[19] D.M. Small. The Physical Chemistry of Lipids. Plenum., N.Y.-London. (1986). $420 \mathrm{p}$

Редактор К.В. Емиев 